\title{
Array Signal Processing for Maximum Likelihood Direction-of-Arrival Estimation
}

\author{
Minghui $\mathrm{Li}^{1 *}$, Yilong $\mathrm{Lu}^{2}$ and $\mathrm{Bo} \mathrm{He}^{3}$
}

${ }^{1}$ School of Engineering, University of Glasgow, Glasgow, United Kingdom

${ }^{2}$ School of Electrical and Electronic Engineering, Nanyang Technological University, Singapore

${ }^{3}$ School of Information Science and Engineering, Ocean University of China, Qingdao, China

\begin{abstract}
Emitter Direction-of-Arrival (DOA) estimation is a fundamental problem in a variety of applications including radar sonar, and wireless communications. The research has received considerable attention in literature and numerous methods have been proposed. Maximum Likelihood (ML) is a nearly optimal technique producing superior estimates compared to other methods especially in unfavorable conditions, and thus is of significant practical interest. This paper discusses in details the techniques for ML DOA estimation in either white Gaussian noise or unknown noise environment. Their performances are analyzed and compared, and evaluated against the theoretical lower bounds.
\end{abstract}

Keywords: Signal processing; Direction-of-Arrival estimation; Maximum likelihood

\section{Introduction}

Estimation of the emitters' directions with an antenna array, or Direction-of-Arrival (DOA) estimation, is an essential problem in a large variety of applications such as radar, sonar, mobile communications, and seismic exploration, because it is a major method for location determination. In wireless communications, DOA estimation may significantly improve communication efficiency and network capacity, support and enhance location-aided routing, dynamic network planning and different types of location-related services and applications. In radar and sonar, accurate target localization is a fundamental objective. The problem has been an active research area for decades, and many high resolution methods have been investigated, such as Multiple Signal Classification (MUSIC), Estimation of Signal Parameters via Rotational Invariance Technique (ESPRIT), Minimum Variance Distortionless Response (MVDR), and Model of Direction Estimation (MODE). An introduction to DOA estimation and array processing can be found, e.g., in $\mathrm{Li}$, et al. [1] and the references therein.

Maximum Likelihood (ML) represents an important category of DOA estimators that determines source DOAs by maximizing the loglikelihood function, which signifies that signals from those directions are most likely to cause occurrence of the given samples. ML produces superior estimates compared to other methods, especially in unfavorable conditions involving low SNR, short data samples, highly correlated or coherent sources, and small array apertures, and thus is of practical interest. It can be used as a caliber to evaluate the performance of other methods. ML DOA estimation has received considerable attention in literature [2-39]. GA-ML is presented in the study of $\mathrm{Li}$ and $\mathrm{Lu}$ [2], which utilizes an enhanced Genetic Algorithm (GA) to find the exact solutions to the highly nonlinear and multi-modal likelihood function. With the newly introduced features, carefully selected operators and fine-tuned parameters, GA-ML achieves fast global convergence. In order to accurately resolve closely spaced sources, a resampling scheme is investigated in [3], where a single data set is resampled to create multiple snapshots in parallel. The computational burden of DOA estimation with large arrays is often prohibitively extensive. To address this challenge, a robust solution for data reduction (and thus computation reduction) in array processing is presented in the study of $\mathrm{Li}$ and $\mathrm{Lu}[4,5]$. In many practical scenarios, the antenna arrays are not well calibrated, due to amplitude and phase mismatch of the receivers, inaccurate sensor locations, and imperfect sensor gain or phase characteristics, or a combination of these effects; DOA estimation using partially calibrated arrays is addressed in in the study of $\mathrm{Li}$ and $\mathrm{Lu}[6,7]$. In real radar or communication systems, the noise often tends to become correlated along the array if the external noise and the coupling between antenna elements cannot be ignored. As a result, the noise covariance is generally unknown and may change slowly with time. Algorithms for DOA estimation without the statistical knowledge of the noise environment are discussed in the study of $\mathrm{Li}$ and $\mathrm{Lu}$ [8]. The DOA processing techniques have been investigated in a variety of applications including radar [9-11], mobile communications [1214], wireless sensor networks [15-18], and ultrasound non-destructive evaluation and imaging [19-24].

In this paper, we focus on maximum likelihood DOA estimation. Section 2 presents the general array data model. Section 3 and 4 discuss in details the techniques for DOA estimation in white Gaussian noise and colored noise fields, respectively. Simulation results are given in Section 5.

\section{Data Model and Problem Formulation}

Consider a general scenario of an array of $M$ elements arranged in an arbitrary geometry immersed in the far field (planar wave) of $N$ point sources at unknown locations. To simplify the exposition, our discussion is confined to azimuth-only systems, i.e., the sensors and signals are assumed to be co-planar. However, the data model and algorithms presented here are general and the extension to azimuthand-elevation systems is straightforward.

*Corresponding author: Minghui Li, School of Engineering, University of Glasgow, Glasgow, United Kingdom, Tel: 44-141-548-2463; E-mail: minghui.li@ieee.org

Received August 28, 2013; Accepted October 24, 2013; Published October 28 2013

Citation: Li M, Lu Y, He B (2013) Array Signal Processing for Maximum Likelihood Direction-of-Arrival Estimation. J Electr Electron Syst 3: 117. doi:10.4172/23320796.1000117

Copyright: (C) $2013 \mathrm{Li} \mathrm{M}$, et al. This is an open-access article distributed under the terms of the Creative Commons Attribution License, which permits unrestricted use, distribution, and reproduction in any medium, provided the original author and source are credited. 
Citation: Li M, Lu Y, He B (2013) Array Signal Processing for Maximum Likelihood Direction-of-Arrival Estimation. J Electr Electron Syst 3: 117. doi:10.4172/2332-0796.1000117

A standard data model for the problem of DOA estimation is:

$\mathrm{y}(t)=\mathrm{A}(\theta) \mathrm{s}(t)+\mathrm{n}(t), t=1,2, \ldots, L$,

where $y(t)$ is the array output, $s(t)$ is the unknown vector of signal waveforms, $\mathrm{n}(t)$ is an immeasurable noise process, $L$ denotes the number of data samples (snapshots). The matrix $A(\theta)$ has the following special structure:

$$
\mathrm{A}(\theta)=\left[\mathrm{a}\left(\theta_{1}\right) \ldots \mathrm{a}\left(\theta_{\mathrm{N}}\right)\right]
$$

where $\mathrm{a}(\theta)$ is the so-called steering vector that describes a mapping between DOA and array response, and $\theta=\left\{\theta_{1}, \ldots, \theta_{\mathrm{N}}\right\}$ are parameters of interest or DOAs. The exact form of $\mathrm{a}(\theta)$ depends on the array geometry, and is not specified in this section.

In most literature, the number of sources $N$ is assumed to be known (given or estimated). We also assume that the number of sensors is greater than the number of sources, $M>N$, to guarantee the uniqueness of DOA estimation [28]. Assuming that the noise and signals are independent in (1), the data covariance matrix is given by

$$
\mathrm{R}=E\left\{\mathrm{y}(t) \mathrm{y}^{H}(t)\right\}=\mathrm{APA}^{H}+\mathrm{Q} .
$$

where $E[\bullet]$ stands for the expectation operator, $(\bullet)^{H}$ denotes the conjugate transpose, $\mathrm{P}=E\left\{\mathrm{~s}(t) \mathrm{s}^{H}(\mathrm{~s})\right\}$, and $\mathrm{Q}=E\left\{\mathrm{n}(t) \mathrm{n}^{H}(\mathrm{~s})\right\}$. When only $L$ data samples are available, an unbiased estimate of the covariance matrix can be obtained using an averaging scheme

$$
\hat{\mathrm{R}}=\frac{1}{L} \sum_{t=1}^{L} \mathrm{y}(t) \mathrm{y}^{H}(t) \text {. }
$$

The problem addressed herein is the estimation of $\theta$ (and if necessary, along with the parameters in $\mathrm{P}$ and Q), from a batch of $L$ measurements $\mathrm{y}(1), \ldots, \mathrm{y}(L)$.

\section{DOA Estimation in White Gaussian Noise}

The noise in most receiving systems consists of internal noise and external noise. The internal noise is produced by the electronic device and includes thermal noise and weak versions of other signals in the system, such as clocks and local oscillators. The external noise is defined as an unwanted random signal that is intercepted by the sensors. If the system is designed well, so that there is no coupling between the antennas, and it is assumed that the thermal noise is dominant, a good model for the noise is white Gaussian noise with covariance being a scaled identity matrix in (3),

$$
\mathrm{Q}=\sigma_{n}^{2} \mathrm{I}
$$

where $\sigma_{n}^{2}$ is the noise power, and $\mathbf{I}$ is an identity matrix. This model assumes that the noise intensity is the same in all sensors and that there is no correlation between the noises at any two elements. Regarding the source signals, there are two types of models in current use: conditional model, which assumes the signals to be deterministic and unknown sequences; and unconditional model, which assumes the signals to be random. These two models lead to different ML methods, termed CML and UML respectively [29].

\section{Conditional Maximum Likelihood (CML) estimator}

Assume the signals $\mathrm{s}(t)$ to be deterministic and unknown sequences, and the noise $\mathrm{n}(t)$ to be stationary zero-mean white Gaussian process, $E\left\{\mathrm{n}(t) \mathrm{n}^{H}(t)\right\}=\sigma_{n}^{2} \mathrm{I}$, therefore $\mathrm{y}(t) \sim G\left(\operatorname{As}(t), \sigma_{n}^{2} \mathrm{I}\right)$, where $G(\bullet)$ denotes Gaussian distribution.

The likelihood function of the snapshots $\mathrm{y}(1), \ldots, \mathrm{y}(L)$ is given by

$$
L(\mathrm{y}(1), \cdots, \mathrm{y}(L))=\prod_{t=1}^{L} \frac{1}{\pi \operatorname{det}\left[\sigma_{n}^{2} \mathrm{I}\right]} \exp \left(-\frac{1}{\sigma_{n}^{2}}|\mathrm{y}(t)-\operatorname{As}(t)|^{2}\right),
$$

where $\operatorname{det}[\bullet]$ stands for the determinant. Thus, the log-likelihood function is

$$
\ln L=-L \ln \pi-M L \ln \sigma_{n}^{2}-\frac{1}{\sigma_{n}^{2}} \sum_{t=1}^{L}|\mathrm{y}(t)-\operatorname{As}(t)|^{2} .
$$

In (7), $\sigma_{n}^{2}, \theta$ (in $\left.\mathrm{A}(\theta)\right)$ and $\mathrm{s}(t)$ are unknown parameters.

Firstly, we fix $\theta$ and $s(t)$, and calculate the derivatives of (7) with respect to $\sigma_{n}^{2}$,

$\frac{\partial \ln L}{\partial \sigma_{n}^{2}}=-\frac{M L}{\sigma_{n}^{2}}+\frac{1}{\sigma_{n}^{4}} \sum_{t=1}^{L}|\mathrm{y}(t)-\operatorname{As}(t)|^{2}$.

We then get,

$\sigma_{n}^{2}=\frac{1}{M L} \sum_{t=1}^{L}|\mathrm{y}(t)-\operatorname{As}(t)|^{2}$.

Secondly, fixing $\sigma_{n}^{2}$ and $\theta$, we calculate the derivatives of (7) with respect to $\mathrm{s}(t)$

$\frac{\partial \ln L}{\partial \mathrm{s}(t)}=\frac{2}{\sigma_{n}^{2}} \mathrm{~A}^{H}[\mathrm{y}(t)-\operatorname{As}(t)]$,

it arrives at

$\mathrm{s}(t)=\left(\mathrm{A}^{H} \mathrm{~A}\right)^{-1} \mathrm{~A}^{H} \mathrm{y}(t)$

Finally, substituting (9) and (11) back into (7), we obtain the following maximization problem,

$\arg \max _{\theta}\left\{-M L \ln \frac{1}{M L} \sum_{t=1}^{L}\left|\mathrm{y}(t)-\mathrm{A}\left(\mathrm{A}^{H} \mathrm{~A}\right)^{-1} \mathrm{~A}^{H} \mathrm{y}(t)\right|^{2}\right\}$,

which is equivalent to the following minimization problem

$$
\arg \min _{\theta}\left\{\sum_{t=1}^{L}\left|\left[\mathrm{I}-\mathrm{A}\left(\mathrm{A}^{H} \mathrm{~A}\right)^{-1} \mathrm{~A}^{H}\right] \mathrm{y}(t)\right|^{2}\right\} \text {, }
$$

or in a different form,

$$
f_{C M L}(\theta)=\arg \min _{\theta}\left\{\operatorname{tr}\left[\left(\mathrm{I}-\mathrm{A}\left(\mathrm{A}^{H} \mathrm{~A}\right)^{-1} \mathrm{~A}^{H}\right) \hat{\mathrm{R}}\right]\right\},
$$

where $\operatorname{tr}[\cdot]$ denotes the trace, and $\hat{\mathrm{R}}$ is the sample covariance matrix as in (4).

\section{Unconditional Maximum Likelihood (UML) estimator}

If we assume that both the signals and the noise are stationary, temporally white, zero-mean complex Gaussian random processes with second-order moments satisfying (3) and (5), following a similar derivation procedure, we may conclude that the UML estimator is given by minimizing (15)

$$
f_{U M L}(\theta)=\ln \operatorname{det}\left[\mathrm{A} \tilde{P} A^{H}+\tilde{q} \mathrm{I}\right] \text {, }
$$

where

$$
\begin{aligned}
& \tilde{\mathrm{P}}=\left(\mathrm{A}^{H} \mathrm{~A}\right)^{-1} \mathrm{~A}^{H} \hat{\mathrm{R}} \mathrm{A}\left(\mathrm{A}^{H} \mathrm{~A}\right)^{-1}-\tilde{q}\left(\mathrm{~A}^{H} \mathrm{~A}\right)^{-1}, \\
& \tilde{q}=\frac{1}{M-N} \operatorname{tr}\left\{\left[\mathrm{I}-\mathrm{A}\left(\mathrm{A}^{H} \mathrm{~A}\right)^{-1} \mathrm{~A}^{H}\right] \hat{\mathrm{R}}\right\},
\end{aligned}
$$

and $\ln [\bullet]$ denotes the natural logarithm.

Literature [29] demonstrates that for uncorrelated sources, the statistical performances of CML and UML are similar; while for highly correlated or coherent sources, UML is significantly superior. For UML, the stochastic Cramer-Rao Bound (CRB) can be achieved as $N \rightarrow \infty$, or $S N R \rightarrow \infty$; while for CML, the corresponding bound cannot be attained if $M<\infty$, even though $N \rightarrow \infty$, or $S N R \rightarrow \infty$. 
Citation: Li M, Lu Y, He B (2013) Array Signal Processing for Maximum Likelihood Direction-of-Arrival Estimation. J Electr Electron Syst 3: 117. doi:10.4172/2332-0796.1000117

Page 3 of 5

The optimization of the likelihood functions (14) and (15) is a nonlinear optimization problem. In the absence of a closed form solution, it requires iterative schemes for solutions. A couple of such schemes have been proposed for solving this problem, such as alternating projection [30], simulated annealing [31], expectation maximization [32], and data supported grid search [33]. A rapid technique [2] using an enhanced genetic algorithm is presented for solutions in general cases.

\section{DOA Estimation in Unknown Noise Environment}

In this section, we assume the noise covariance $\mathbf{Q}$ to be completely unknown, except for the fact that it is a Hermitian positive definite matrix. Under the assumption of additive Gaussian noise and Gaussian distributed signals, the normalized (with $L$ ) negative log-likelihood function of the data vectors takes the form (ignoring the parameter independent terms) [34]

$$
f(\theta, \mathrm{P}, \mathrm{Q})=\ln \operatorname{det}[\mathrm{R}]+\operatorname{tr}\left[\mathrm{R}^{-1} \hat{\mathrm{R}}\right],
$$

where $\ln \operatorname{det}[\cdot]$ denotes the natural logarithm of the determinant.

\section{ML estimation based on parametric noise covariance}

Based on a Fourier series expansion of the spatial noise power density function, the noise covariance $\mathbf{Q}$ is assumed to be modeled by the following linear parameterization:

$$
\mathrm{Q}(\eta)=\sum_{j=1}^{J} \eta_{j} \Sigma_{j}
$$

where $\eta=\left[\eta_{1}, \ldots, \eta_{J}\right]^{T}$ is a vector of unknown noise Fourier coefficients, $\Sigma_{j}$ is a known function of the array geometry given by

$\Sigma_{j}=\left\{\begin{array}{cc}\bar{\Sigma}_{(j-1) / 2} & j \text { odd } \\ \tilde{\Sigma}_{j / 2} & j \text { even }\end{array}\right.$

where

$\bar{\Sigma}_{l} \int_{-\pi}^{\pi} \mathrm{a}(\theta) \mathrm{a}^{H}(\theta) \cos (l \theta) d \theta$
$\tilde{\Sigma}_{l} \int_{-\pi}^{\pi} \mathrm{a}(\theta) \mathrm{a}^{H}(\theta) \sin (l \theta) d \theta$

$l=0,1,2, \cdots$. Similar noise models have appeared in the literature [35-37]. We assume that the number of signals $N$ and the number of noise parameters $J$ are known or have been estimated [36].

By solving for $\mathrm{P}$ in terms of $\theta$ and $\mathrm{Q}(\eta)$ and then substituting back to (16), similar to the derivation in Section 3.1, we get an Exact ML (EML) function that depends on both $\theta$ and $\eta$ [8]

$$
f_{E M L}(\theta, \eta)=\ln \operatorname{det}[\mathrm{Q}]+\ln \operatorname{det}[\mathrm{G} \overline{\mathrm{R}} \mathrm{G}+\mathrm{H}]+\operatorname{tr}[\mathrm{H} \overline{\mathrm{R}}],
$$

Where

$$
\begin{aligned}
& \overline{\mathrm{A}}=\mathrm{Q}^{-1 / 2} \mathrm{~A} \\
& \mathrm{G}=\overline{\mathrm{A}}\left(\overline{\mathrm{A}}^{H} \overline{\mathrm{A}}\right)^{-1} \overline{\mathrm{A}}^{H} \\
& \overline{\mathrm{R}}=\mathrm{Q}^{-1 / 2} \hat{\mathrm{R}} \mathrm{Q}^{-1 / 2} \\
& \mathrm{H}=\mathrm{I}-\mathrm{G}
\end{aligned}
$$

The ML estimates of $\theta$ and $\eta$ are obtained by minimizing (19). Further derivation of a function merely depending on the DOAs seems impossible. However, using the large sample assumption and least square criteria, we can get the following approximation of (19) [8]

$$
f_{A M L}(\theta)=\left\|\Pi\left(\mathrm{I}-\mathrm{B}\left(\mathrm{B}^{H} \Pi \mathrm{B}\right)^{-1} \mathrm{~B}^{H} \Pi\right) \mathrm{d}\right\|^{2},
$$

where

$$
\begin{aligned}
& \mathrm{d}=\operatorname{vec}\{\hat{\mathrm{R}}\} \\
& \mathrm{B}=\mathrm{A}^{*} \otimes \mathrm{A} \\
& \Gamma=\left[\operatorname{vec}\left\{\Sigma_{1}\right\}, \ldots \operatorname{vec}\left\{\Sigma_{\}}\right\}\right] \\
& \Pi=\mathrm{I}-\Gamma\left(\Gamma^{H} \Gamma\right)^{-1} \Gamma^{H}
\end{aligned}
$$

$\operatorname{vec}\{\bullet\}$ is a concatenation of the columns of the bracketed matrix, $\otimes$ denotes Kronecker product, and $\|\cdot\|$ stands for Euclidean norm.

\section{ML estimation based on parameterized signals}

In this method, the signals are assumed to be linear combinations of $p$ known basis functions:

$$
\mathrm{s}(t)=\mathrm{T}^{H} \mathrm{~b}(t),
$$

where $\mathrm{T}$ is an unknown $p \times N$ matrix, and the $p$-vector $\mathrm{b}(t)$ contains the basic functions that are uncorrelated with the noise. Possible applications where this assumption is reasonable include active radar or sonar, and mobile communications [38].

The exact ML estimates of source parameters are the minimizing argument of the following function [8]

$$
f_{\mathrm{EML}}(\theta, \varphi)=\ln \operatorname{det}\left[\mathrm{I}+\Delta_{0}\right],
$$

where

$$
\begin{aligned}
& \Delta_{0}=\hat{\mathrm{R}}_{b b}^{-1 / 2} \hat{\mathrm{R}}_{y b}^{H} \hat{\mathrm{W}}^{-1 / 2} \hat{\Pi}^{\perp} \hat{\mathrm{W}}^{-1 / 2} \hat{\mathrm{R}}_{y b} \hat{\mathrm{R}}_{b b}^{-1 / 2} \\
& \hat{\Pi}^{\perp}=\mathrm{I}-\hat{\mathrm{W}}^{-1 / 2} \mathrm{~A}\left(\mathrm{~A}^{H} \hat{\mathrm{W}}^{-1} \mathrm{~A}\right)^{-1} \mathrm{~A}^{H} \hat{\mathrm{W}}^{-1 / 2} \\
& \hat{\mathrm{W}}=\hat{\mathrm{R}}-\tilde{\mathrm{R}}_{y b} \tilde{\mathrm{R}}_{y b}^{H} \\
& \hat{\mathrm{R}}_{y b}=\frac{1}{L} \sum_{t=1}^{L} \mathrm{y}(t) \mathrm{b}^{H}(t) \\
& \hat{\mathrm{R}}_{b b}=\frac{1}{L} \sum_{t=1}^{L} \mathrm{~b}(t) \mathrm{b}^{H}(t) \\
& \tilde{\mathrm{R}}_{y b}=\hat{\mathrm{R}}_{y b} \hat{\mathrm{R}}_{b b}^{-1 / 2} .
\end{aligned}
$$

$\varphi$ contains the unknown parameters in basis functions $\mathrm{b}(t)$ Provided $\mathrm{b}(t)$ is completely known and the samples are large enough, (22) can be simplified. Since $\Delta_{0}$ is small (of order $O(1 / L)$ ) for large $L$ and near the true $\theta$, we may approximate $\ln \operatorname{det}\left[\mathrm{I}+\Delta_{0}\right]$ by the first term of the Taylor series expansion

$$
\ln \operatorname{det}\left[\mathrm{I}+\Delta_{0}\right]=\operatorname{tr}\left[\Delta_{0}\right]-\frac{1}{2} \operatorname{tr}\left[\Delta_{0}^{2}\right]+\cdots .
$$

Hence, the approximate ML (AML) function merely depending on $\theta$ can be obtained as follows [8]

$$
f_{A M L}(\theta)=\operatorname{tr}\left[\hat{\Pi}^{\perp} \hat{\mathrm{W}}^{-1 / 2} \hat{\mathrm{R}}_{y b} \hat{\mathrm{R}}_{b b}^{-1} \hat{\mathrm{R}}_{y b}^{H} \hat{\mathrm{W}}^{-1 / 2}\right] .
$$

It should be noted that, the AML estimator has the form of a standard signal subspace fitting structure, for which several minimization techniques have been proposed, for instance alternating projection [30] and the modified Newton technique [39].

\section{Simulation Studies}

The main performance indices for a DOA estimator include bias, variance, and resolution probability, which are complicated functions of source SNR, the number of snapshots, number and directions of sources, and the array geometry. A poor estimate generally results from using shorter snapshots and sources with lower SNR. Even though bias and variance both play important roles in direction estimation, the 
Citation: Li M, Lu Y, He B (2013) Array Signal Processing for Maximum Likelihood Direction-of-Arrival Estimation. J Electr Electron Syst 3: 117. doi:10.4172/2332-0796.1000117

Page 4 of 5

effect of bias is more critical in the threshold region. The variance is often evaluated against the CRB, which provides a lower bound on the covariance matrix of any unbiased estimators, and is expected to be a good performance predictor for large samples.

In the follows, we present two numerical examples to compare the performances of ML estimators with other popular techniques including MUSIC, ESPRIT and MVDR, and evaluate them against the CRB. Each simulated point is calculated from 500 independent MonteCarlo trials. The performances of those methods are compared at two aspects: 1) DOA estimation Root-Mean-Squared Error (RMSE), and 2) resolution probability.

An 8-element Uniform Linear Array (ULA) with half-wavelength element spacing is considered in Example 1. Two equal-power correlated signals with the correlation factor $\gamma=0.8$ are assumed to illuminate the array from $61^{\circ}$ and $64^{\circ}$ relative to the end-fire. The number of snapshots is 40 , and the SNR is varied. The noise is assumed to be white Gaussian noise.

Figure 1 depicts the DOA estimation RMSE obtained by UML, ESPRIT, MUSIC and MVDR, and compares them with the CRB. Figure 2 shows the resolution probabilities for the same methods. As can be seen from Figure 1 and Figure 2, UML demonstrates much better performance than the other techniques as a whole, producing more accurate estimates in terms of RMSE, and better source resolving

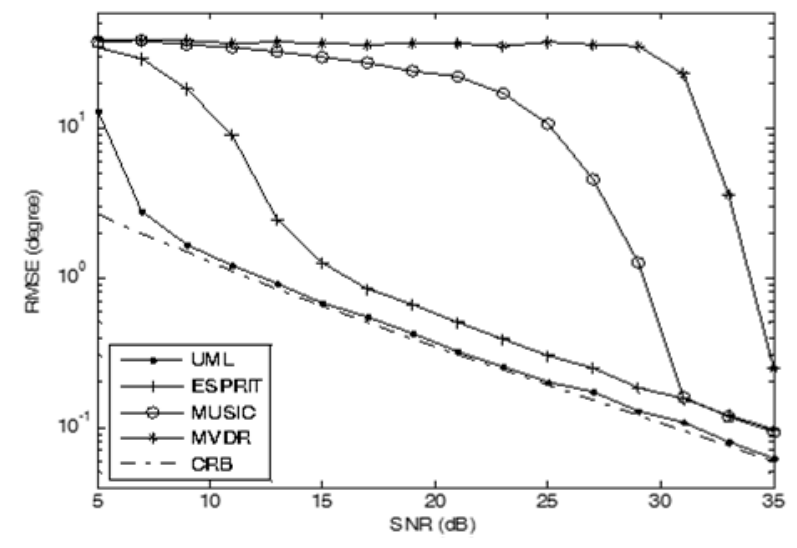

Figure 1: DOA estimation RMSE versus SNR.

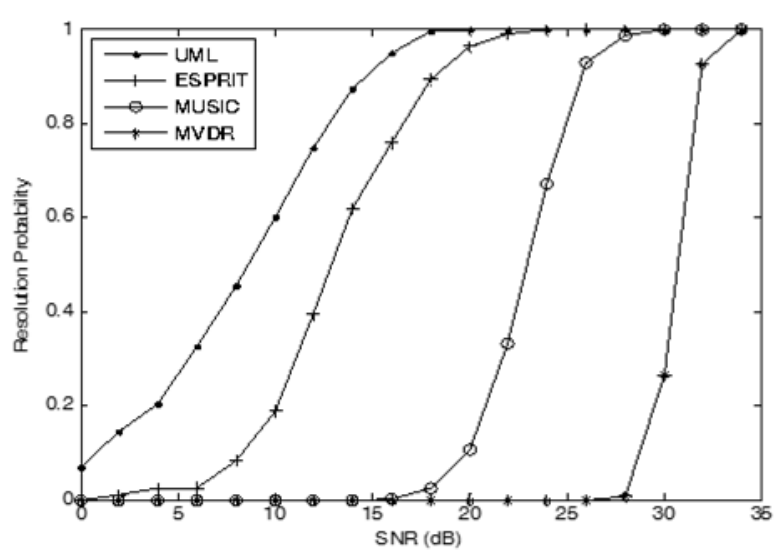

Figure 2: DOA resolution probability versus SNR.

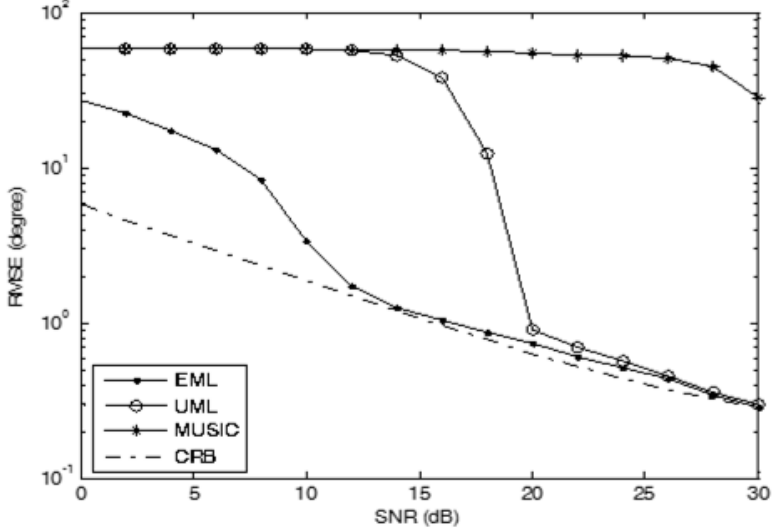

Figure 3: DOA estimation RMSE versus SNR.

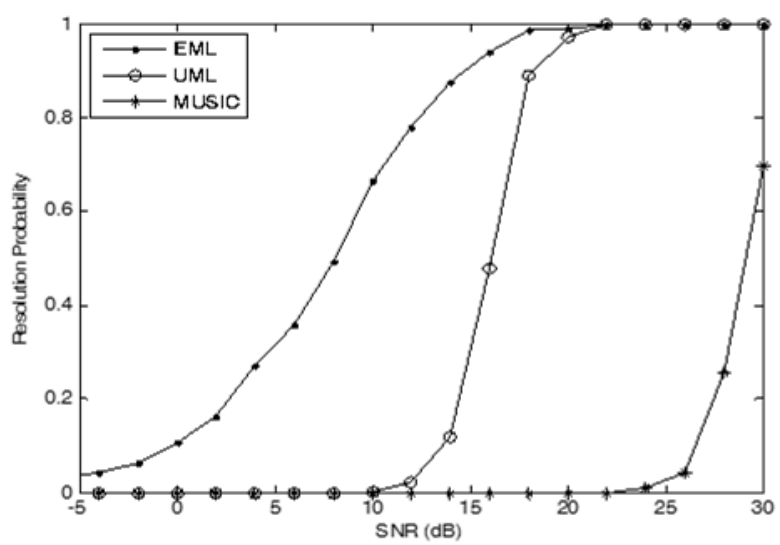

Figure 4: DOA resolution probability versus SNR.

power in terms of resolution probabilities. UML asymptotically attains the CRB when SNR gets higher. ESPRIT performs better than MUSIC and MVDR in the cases of correlated sources, and MVDR demonstrates the strongest threshold effect when SNR is low.

Example 2 is provided to demonstrate the performance of the ML estimator in correlated noise fields, and evaluate it against MUSIC and UML. We consider the data model with the noise covariance being a linear combination of known matrices as in (17). $J=3$, and the noise parameters are $\eta=[1,1 / 4,1 / 9]$. Assume that two equal-power correlated signals with the correlation factor $r=0.95$ impinge on a four-element ULA from $90^{\circ}$ and $95^{\circ}$ relative to the end-fire. The number of snapshots is 80 .

Figure 3 depicts the DOA estimation RMSE obtained using EML (19), MUSIC and UML as a function of SNR, and compares them with the corresponding CRB. Figure 4 shows the resolution probabilities for the same methods. As can be seen from Figure 3 and Figure 4, the EML technique that takes the noise correlation into account yields significantly superior performance over MUSIC and UML as a whole, by demonstrating lower estimation RMSE and higher resolution probabilities. EML produces excellent estimates with RMSE approaching and asymptotically attaining the theoretic lower bound. On the other hand, as a standard high-resolution method in white Gaussian noise, MUSIC fails almost in the whole SNR range. Although 
Citation: Li M, Lu Y, He B (2013) Array Signal Processing for Maximum Likelihood Direction-of-Arrival Estimation. J Electr Electron Syst 3: 117. doi:10.4172/2332-0796.1000117

Page 5 of 5

UML is an optimal technique in white noise, it completely fails when SNR is lower than $15 \mathrm{~dB}$ and only produces acceptable estimates in high SNR region. The results can be explained by the fact that UML and MUSIC are sensitive to modeling errors due to noise correlation. It is worth noting that the advantages of EML over the other techniques are more prominent when SNR is low, and the benefits can be extended to other unfavorable scenarios involving short samples, clustered sources, and small array apertures.

\section{Conclusions}

This paper explains how DOA estimation can be obtained using antenna arrays, and discusses the data model and various signal processing algorithms. ML DOA estimation is a nearly optimal technique, which produces superior estimates compared to other methods. A detailed treatment of the ML algorithms for DOA estimation in white Gaussian noise and colored noise environment has been provided by including the description, analysis and performance evaluation with numerical simulations.

\section{References}

1. Li MH, Lu YL, Chen HH, Wang B, Chen IM (2009) Angle-of-arrival (AOA) estimation in wireless networks. Wireless networks: Research, technology and applications, Nova Science Publishers, Inc., New York, USA, 5: 135-164.

2. Li MH, Lu YL (2007) A refined genetic algorithm for accurate and reliable DOA estimation with a sensor array. Wireless Pers Commun 43: 533-547.

3. Li MH, Lu YL (2004) Improving the performance of GA-ML DOA estimator with a resampling scheme. Signal Process 84: 1813-1822.

4. Li MH, Lu YL (2006) Dimension reduction for array processing with robust interference cancellation. IEEE T Aero Elec Sys 42: 103-112.

5. Li MH, Lu YL (2005) Null-steering beamspace transformation design for robus data reduction. Proceedings of 13th European Signal Processing Conference.

6. Li MH, Lu YL (2009) Source bearing and steering-vector estimation using partially calibrated arrays. IEEE T Aero Elec Sys 45: 1361-1372.

7. Li MH, Lu YL (2007) Maximum likelihood processing for arrays with partially unknown sensor gains and phases. Proceedings of 7 th International Conference on Intelligent Transportation Systems Telecommunications.

8. Li MH, Lu YL (2008) Maximum likelihood DOA estimation in unknown colored noise fields. IEEE T Aero Elec Sys 44: 1079-1090.

9. Haykin S, Litva J, Shepherd TJ (1993) Radar Array Processing. SpringerVerlag, New York, USA.

10. Yoke LS, Agatonovic M, Zwick T (2012) Neural network based direction of arrival estimation for a MIMO OFDM radar. Proceedings of 9th European Radar Conference.

11. Li MH, Lu YL (2002) Genetic algorithm based maximum likelihood DOA estimation. Proceedings of Radar Conference.

12. Abdalla MM, Abuitbel MB, Hassan MA (2003) Performance evaluation of direction of arrival estimation using MUSIC and ESPRIT algorithms for mobile communication systems. Proceedings of 6th Wireless and Mobile Networking Conference.

13. Madyastha R, Aazhang B (1997) Delay and DOA estimation in CDMA communication systems via maximum likelihood techniques. Proceedings of IEEE International Symposium on Information Theory.

14. Li MH, Wang B, Lu YL, Zhou MT, Chen IM (2010) Smart antenna in intelligent transportation systems. Wireless Technologies in Intelligent Transportation Systems, Nova Science Publishers, Inc. New York.

15. Li MH, Lu YL, Wee L (2006) Target detection and identification with a heterogeneous sensor network by strategic resource allocation and coordination. Proceedings of 6 th International Conference on Intelligent Transportation Systems Telecommunications.

16. Wang B, Li MH, Lim HB, Ma D, Fu C (2009) Energy efficient information processing in wireless sensor networks. Guide to Wireless Sensor Networks Springer, London, UK.

17. Li MH, Lu YL (2007) Optimal direction finding in unknown noise environments using antenna arrays in wireless sensor networks. Proceedings of 7th International Conference on Intelligent Transportation Systems Telecommunications

18. Li MH, Lu YL (2008) Angle-of-arrival estimation for localization and communication in wireless networks. Proceedings of 16th European Signal Processing Conference.

19. Li MH, Hayward G (2012) Ultrasound nondestructive evaluation (NDE) imaging with transducer arrays and adaptive processing. Sensors 12: 42-54.

20. Gongzhang R, Li MH, Lardner T, Gachagan A, Hayward G (2012) Robust defect detection in ultrasonic nondestructive evaluation (NDE) of difficult materials. Proceedings of 2012 IEEE International Ultrasonics Symposium.

21. Lardner T, Li MH, Gongzhang R, Gachagan A (2012) A new speckle noise suppression technique using cross-correlation of array sub-apertures in ultrasonic NDE of coarse grain materials. Proceedings of Review of Progress in Quantitative Nondestructive Evaluation (QNDE).

22. Li MH, Hayward G, He B (2011) Adaptive array processing for ultrasonic nondestructive evaluation. Proceedings of 2011 IEEE International Ultrasonics Symposium

23. Li MH, McGuire M, Ho KS, Hayward G (2010) Array element failure correction for robust ultrasound beamforming and imaging. Proceedings of 2010 IEEE International Ultrasonics Symposium.

24. He B, Liang Y, Feng X, Nian R, Yan T, et al. (2012) AUV SLAM and experiments using a mechanical scanning forward-looking sonar. Sensors 12: 9386-9410.

25. Li K, Lu YL, Li MH (2005) Approximate formulas for lateral electromagnetic pulses from a horizontal electric dipole on the surface of one-dimensionally anisotropic medium. IEEE T Antenn Propag 53: 933-937.

26. Li MH, Ho KS, Hayward G (2009) Beamspace transformation for data reduction using genetic algorithms. Proceedings of IEEE International Ultrasonics Symposium

27. Li MH, Ho KS, Hayward G (2010) Accurate angle-of-arrival measurement using particle swarm optimization. Wireless Sensor Network 2: 358-364.

28. Wax M, Ziskind I (1989) On unique localization of multiple sources by passive sensor arrays. IEEE T Acoust Speech 37: 996-1000.

29. Stoica P, Nehorai A (1990) Performance study of conditional and unconditional direction-of-arrival estimation. IEEE T Acoust Speech 38: 1783-1795.

30. Ziskind I, Wax M (1988) Maximum likelihood localization of multiple sources by alternating projection. IEEE T Acoust Speech 36: 1553-1560.

31. Sharman K (1988) Maximum likelihood estimation by simulated annealing Proceedings of ICASSP-88.

32. Miller M, Fuhrmann D (1990) Maximum likelihood narrow-band direction finding and the EM algorithm. IEEE T Acoust Speech 38: 1560-1577.

33. Stoica P, Gershman A (1999) Maximum-likelihood DOA estimation by datasupported grid search. IEEE Signal Proc Let 6: 273-275.

34. Ottersten B, Viberg M, Stoica P, Nehorai A (1993) Exact and large sample maximum likelihood techniques. Radar Array Processing, Springer-Verlag, New York, USA.

35. Fuchs J (1992) Estimation of the number of signals in the presence of unknown correlated sensor noise. IEEE T Signal Proces 40: 1053-1061.

36. Vanpoucke F, Paulraj A (1995) A harmonic noise model for direction finding in colored ambient noise. IEEE Signal Proc Let 2: 135-137.

37. Göransson B, Ottersten B (1999) Direction estimation in partially unknown noise fields. IEEE T Signal Proces 47: 2375-2385.

38. Viberg M, Stoica P, Ottersten B (1997) Maximum likelihood array processing in spatially correlated noise fields using parameterized signals. IEEE T Signa Proces 45: 996-1004.

39. Ye H, DeGroat RD (1995) Maximum likelihood DOA estimation and asymptotic Cramér-Rao bounds for additive unknown colored noise. IEEE T Signal Proces 43: 938-949. 